\title{
PERCEPÇÃo dOS PROFISSIONAIS DE SAÚdE QUANTO AO EFEITO DE UM GRUPO DE APOIO À SAÚdE MENTAL NA ATENÇÃo PRIMÁria À SAÚdE
}

\author{
Perception of health professionals about the effect of \\ a mental health support group in Primary Health Care
}

\author{
Maria Luiza Melo Machado', Ana Paula Ferreira Gomes²
}

1. Psicóloga Residente do Programa de Saúde da Família da SMS de Curitiba/FEAES. Especialista em Psicologia, Habilidades Sociais e Desenvolvimento Humano. ORCID: https://orcid.org/0000-0001-9026-957X.

2. Psicóloga da Secretaria Municipal de Saúde de Curitiba: Tutora e Preceptora da Residência Multiprofissional em Saúde da Família da SMS/FEAES; Docente dos cursos de Medicina e Psicologia das Faculdades Pequeno Príncipe. Mestre em Gestão de Tecnologia e Inovação em Saúde. ORCID: https://orcid.org/0000-00026484-7177.

CONTATO: Maria Luiza Melo Machado | R. Leon Nicolas, 195 | Capão Raso | Curitiba | Paraná | Brasil | CEP 81150-140 | E-mail: mluiza_mm@yahoo.com.br

COMO CITAR Machado MLM, Gomes APF. Percepção dos profissionais de saúde quanto ao efeito de um grupo de apoio à saúde mental na atenção primária à saúde. R. Saúde Públ. 2018 Jul.;1(1):35-46.

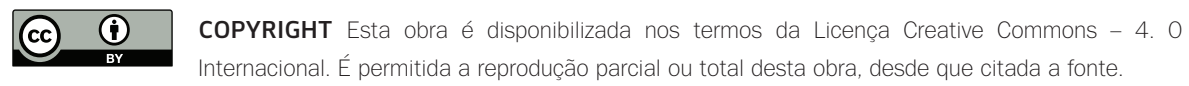

RESUMO A inclusão das ações de saúde mental na Atenção Primária à Saúde atende aos princípios do Sistema Único de Saúde e contribui para o processo da Reforma Psiquiátrica brasileira. Neste contexto, este estudo relata o efeito de um grupo na relação de cuidado com usuários com transtornos mentais em uma Unidade Básica de Saúde de Curitiba. Para investigar seu efeito, levantou-se a percepção dos profissionais envolvidos, a partir da análise de conteúdo. Os relatos dos profissionais foram agrupados em temas e núcleos de discussões; as ocorrências das respostas foram enumeradas (quantitativo) e os conteúdos selecionados foram analisados (qualitativo). Nos resultados, percebeu-se efeito positivo na assistência à saúde mental, destacando-se melhora na autoestima, autonomia e comunicação dos usuários; bem como mudanças de atitude daqueles que prestam cuidado, com quebra de preconceitos e redução de estigmas, proporcionando um melhor acolhimento e compreensão do contexto em que os usuários vivem.

PALAVRAS-CHAVE: Saúde Mental. Atenção Primária à Saúde. Serviços Comunitários de Saúde Mental 
ABSTRACT In Brazil, the inclusion of mental health actions in Primary Health Care Services follows the principles of its Public Health System, and contributes to the process of the Brazilian Psychiatric Reform Policies. Thus, this study reports the effect of a mental health supportive group on the relationship with users in a Basic Health Unit in the city of Curitiba. In order to investigate its effect, the perception of the professionals involved was surveyed through content analysis. The professionals' answers reports were divided into themes and discussion groups; the answers were listed (quantitative) and the selected contents were analyzed (qualitative). The results showed a positive impact on mental health care, with improvement in self-esteem, autonomy and communication of patients being highlighted, as well as changes in the attitude of health care providers, including the reduction of prejudice and stigma, providing better care and understanding of the context in which these patients live.

KEYWORDS: Mental Health. Primary Health Care. Community Mental Health Services

\section{INTRODUÇÃO}

$\mathbf{A}$ s preocupações com a saúde mental durante muitos anos foram marginalizadas, sendo que as pessoas com algum diagnóstico de transtorno mental eram consideradas potencial risco à sociedade'.

Após a Revolução Francesa - que trazia como slogan "Fraternidade, liberdade e igualdade" - os cuidados dos pacientes excluídos da sociedade, começaram a sofrer mudanças'. Neste contexto, Philippe Pinel iniciou uma reformulação dos hospitais psiquiátricos, visando a necessidade de tratamento, defendendo a importância de desacorrentar os alienados e inscrever suas "alienações" na nosografia médica. Para tal, separou os ditos loucos dos demais excluídos (criminosos) com o objetivo de estudálos, propondo o isolamento para conhecer sobre a enfermidade que os loucos eram portadores.

Em meados do século $X X$, foi constatado que, além da exclusão social, os hospitais psiquiátricos e os manicômios utilizavam de práticas violentas. Nesse período foram iniciadas críticas aos hospícios, destacando-se as contribuições de Laing (1960), Cooper (1967), Szasz (1960), Foucault (1954), Deleuze (1968), Guatarri (1966), Caplan
(1980) e Basaglia (1967), e o surgimento de diversas propostas como: a Psiquiatria Preventiva Comunitária Norte-americana de Caplan (1980), nos Estados Unidos; a Psiquiatria de Setor de Bonnafé (1945) e a Psicoterapia Institucional de Oury e Guattari (1966), na França; o modelo de Comunidade Terapêutica de Maxwell Jones (1953). na Inglaterra². Essas iniciativas tinham o intuito de adequar o tratamento a uma ideologia em que a assistência pudesse ter a participação democrática e o bem-estar social a todos os envolvidos no processo do cuidado dos pacientes, emergindo a indispensabilidade de uma Reforma Psiquiátrica³

Uma das principais mudanças no modelo de assistência hospitalar ocorreu em meados de 1940 , na unidade de reabilitação social Belmont Hospital, na Inglaterra, onde foi desenvolvida uma metodologia que rompia com o tratamento exclusivamente individual, propondo uma psiquiatria social que reforçava o envolvimento interpessoal - o uso de métodos grupais - cujo objetivo era considerar a instituição como uma comunidade, na qual se esperava que todos contribuíssem para alcançar a mesma meta: criação de uma organização social 
dotada de propriedade de cura ${ }^{4}$. Neste contexto hospitalar surgiu a designação de comunidade terapêutica, sendo esta a primeira estratégia utilizada para superar o modelo excludente dos manicômios. A lógica desse modelo assistencial era baseada na democracia das relações, participação e papel terapêutico de todos os membros da comunidade, com ênfase na comunicação e no trabalho, como instrumentos essenciais no processo de recuperação dos internos ${ }^{3,5}$.

Em 1961, na Itália, Franco Basaglia, insatisfeito com o sistema psiquiátrico da época - não aceitava o estilo de vida que era ofertado aos internos e os métodos coercitivos e violentos utilizados no tratamento - passou a utilizar medidas que visavam a humanidade do paciente, com o objetivo de resgatar a dignidade e seus direitos ${ }^{3}$.

Basaglia propôs, inicialmente, transformar os hospícios em uma comunidade terapêutica, visando um espaço de cura. Buscou melhorar as condições de hospedaria e o cuidado técnico aos internos. Porém, à medida que se defrontava com a miséria humana criada pelas condições do hospital, percebia que uma simples humanização deste não seria suficiente, eram necessárias transformações profundas tanto no modelo de assistência psiquiátrica quanto nas relações entre a sociedade e a loucura ${ }^{6}$

A partir de um processo de longa resistência, o modelo de tratamento em hospitais e manicômios foi sendo substituído por uma rede territorial de atendimento, na qual faziam parte os serviços de atenção comunitária, a emergência psiquiátrica em hospital geral, as cooperativas de trabalho protegido, centros de convivência e moradias assistidas (chamadas por ele de "gruposapartamento") para os loucos ${ }^{6}$.

A doença mental começou a ser compreendida para além do campo do saber médico - antes resolvida simplesmente por soluções técnicas -. passando a incluir outras dimensões, como: a classe social, na qual a maioria eram pessoas mais carentes; aspectos referentes à natureza jurídica e policial, na manutenção da ordem pública; a não neutralidade da ciência, quando passou a se manifestar contra o saber absoluto da psiquiatria; e, também, o reconhecimento do papel e da função dos profissionais como agentes empoderadores e de controle na instituição ${ }^{5}$.

Em meados de 1970 e 1980, a Organização Mundial da Saúde (OMS) reconheceu o impacto dos problemas de saúde mental e questionou os cuidados exclusivamente na atenção especializada, preconizando a descentralização dos serviços existentes, a integração de serviços psiquiátricos em unidades de cuidados gerais, a formação de cuidadores não especializados e o aumento da participação da comunidade ${ }^{7.8}$.

A partir de 1976, Basaglia desenvolveu uma assistência em saúde mental que, na sua totalidade, era exercida na rede territorial ${ }^{5}$. Influenciando o cenário mundial, preconizava um novo paradigma em saúde, no qual propunha tornar a Atenção Primária à Saúde (APS) em um ponto estratégico da rede de cuidados e da reestruturação de todo o sistema, sendo a responsável por solucionar os principais problemas de saúde da população, inclusive os referentes à saúde mental ${ }^{9}$.

A Reforma Psiquiátrica no Brasil surgiu com o Movimento dos Trabalhadores de Saúde Mental (MTSM), no final da década de 1970, fortemente influenciada pelos princípios e diretrizes defendidos por Basaglia - denunciando a violência nos hospitais e o desrespeito aos direitos humanos ${ }^{10}$. Tal movimento provocou e fortaleceu um discurso humanitário em defesa dos pacientes internados, possibilitando o avanço da luta antimanicomial.

No Brasil, a partir da influência da Reforma Sanitária, essas ideologias foram incorporadas e veiculadas pelo movimento da Reforma Psiquiátrica. A 8 a Conferência Nacional de Saúde de 1986, que teve como consequência a realização, no ano de 1987, da I Conferência Nacional de Saúde Mental ${ }^{11}$. Este movimento desloca o tratamento do atendimento centrado no hospital para um atendimento mais ampliado, que possa contemplar a família, as relações sociais e os vínculos do sujeito que vivencia uma situação de sofrimento ${ }^{12}$. 
A promulgação da Constituição Federal de 1988 é considerada um dos momentos mais importantes para a Reforma Psiquiátrica brasileira, com a criação do Sistema Único de Saúde (SUS). que visa a garantia do acesso à saúde como direito de todos, uma transformação significativa que preconizou inúmeras políticas públicas ${ }^{13}$, inclusive na saúde mental. Na década de 1990, nove estados aprovaram leis referentes à assistência para a saúde mental e o Ministério da Saúde publicou oito portarias nesse período, por exemplo: Portaria/ SNAS n 189, de 19 de novembro de 1991; Portaria/ SNAS n²24, de 29 de janeiro de 1992: Portaria/ SAS n 407, de 30 de junho de 1992: Portaria/SAS $n^{\circ}$ 408, de 30 de dezembro de 1992; Portaria/SAS $n^{\circ} 145$, de 25 de agosto de 1994; Portaria/SAS $n^{\circ}$ 147, de 25 de agosto de $1994^{14}$.

Outro fator importante ocorreu no ano de 1989. com o projeto de lei do deputado Paulo Delgado, que também influenciou as legislações estaduais em saúde mental na década de 1990, que propôs priorizar a reorientação do modelo assistencial no qual o sujeito passa a ser acompanhado dentro da sua comunidade, sendo inserido nas ações de: promoção, prevenção, tratamento e reabilitação em saúde - substituindo o modelo manicomial asilar que apresentava conceitos e práticas baseados no isolamento e na exclusão social do fenômeno da loucura ${ }^{13}$.

Porém, esta lei só veio a ser aprovada em 06 de abril de 2001, ficando conhecida como Lei Paulo Delgado ou Lei $10.216^{15}$. Ambos os processos contribuíram para que houvesse uma ampliação e acessibilidade aos serviços em saúde, além de uma ruptura, embora gradual, na maneira de apreender o sujeito em seu sofrimento ${ }^{13.15}$.

No Sistema Único de Saúde, a APS tem como um de seus princípios possibilitar o primeiro acesso das pessoas ao sistema, inclusive daquelas que demandam um cuidado em saúde mental. Neste nível de atenção, as ações são desenvolvidas longitudinalmente, em um território geograficamente conhecido, possibilitando aos profissionais de saúde uma proximidade para conhecer a história de vida das pessoas e de seus vínculos com a comunidade e local onde moram, bem como com outros elementos dos seus contextos de vida ${ }^{16}$.

Desta forma, o cuidado em saúde mental na Atenção Básica se torna estratégico, tendo em vista o vínculo e a relação estabelecida pelas equipes, através da facilidade de acesso aos usuários e vice-versa. Por estas características, é comum que os profissionais de saúde se deparem a todo o momento com pacientes e situações que envolvem o sofrimento psíquico ${ }^{16}$.

Na APS preconiza-se o cuidado humanizado e contextualizado, levando-se em conta aspectos da integralidade e promoção da saúde mental, por meio da clínica ampliada, porém por vezes as práticas de cuidado reproduzem uma lógica reducionista, influenciada pelo modelo tradicional, biomédico ${ }^{17}$. Deste modo, o sofrimento mental acaba sendo tratado como um sintoma, uma sensação desagradável que pode desencadear alguma disfunção orgânica, desconsiderando os diversos significados que o sofrimento pode ter. Nestas situações, em que para cada queixa há uma conduta, pode ocorrer, de modo geral, a medicalização do sofrimento psíquico, sem a consideração do contexto sociofamiliar do usuário, podendo caracterizar situações iatrogênicas. Há de se considerar, ainda, a influência da ideologia de consumo de medicamentos, por concepções de seus consumidores e prescritores em um diálogo contextualizado pelas condições culturais e socioeconômicas ${ }^{18}$.

Em 24 de janeiro de 2008 é promulgada a Portaria 154/GM, que dispõe sobre a criação dos Núcleos de Apoio à Saúde da Família (NASF). que tem como objetivo ampliar a abrangência e o escopo das ações da APS, bem como ser mais resolutiva, fortalecendo e auxiliando no processo de territorialização e regionalização, contribuindo na inserção de diversas estratégias de prevenção e promoção de saúde19. Desta forma, o NASF agrega profissionais por núcleos de saber, inserindo nas equipes ações de apoio e assistência voltadas à atividade física (profissionais de Educação Física), alimentação (nutricionistas), reabilitação 
(fisioterapeutas e fonoaudiólogo), serviço social (assistentes sociais), saúde mental (psicólogos, psiquiatras e terapeutas ocupacionais), saúde da mulher (ginecologistas/obstetras), saúde da criança (pediatras), homeopatia (médico homeopata), acupuntura (médico acupunturista, fisioterapeuta acupunturista e psicólogo acupunturista), assistência farmacêutica (farmacêuticos) entre outras áreas19,20. Diante da complexidade e dados epidemiológicos, a Portaria 154/GM, recomenda que cada equipe NASF tenha pelo menos 1 (um) profissional da área de saúde mental ${ }^{20}$.

Em 2011, através da Portaria no 3088 de 23 de dezembro de 2011, foi instituída a Rede de Atenção Psicossocial (RAPS), que preconiza a criação, ampliação e articulação de atenção à saúde das pessoas com sofrimento ou transtorno mental e com necessidades decorrentes do uso de crack, álcool e outras drogas ${ }^{21}$. A RAPS é constituída por diversos equipamentos de saúde, com diferentes níveis de complexidade, sendo estes: a Unidade Básica de Saúde; o Centro de Atenção Psicossocial (CAPS); as Unidades de Acolhimento (UA); o Serviço de Residência Terapêutica (SRT); a Atenção de Urgência e Emergência; e leitos em Hospitais Gerais e Psiquiátricos, entre outros; podendo ainda contar com recursos e equipamentos de outros setores (assistência social, educação, segurança, etc.) $)^{16,21}$

Em 21 de dezembro de 2017, foi promulgada a portaria $n^{0}$ 3.588, que altera as portarias de consolidação $n^{\circ} 3$ e $n^{\circ} 6$, de 28 de setembro de 2017, incluindo: ambulatórios psiquiátricos RAPS; a reformulação das internações de saúde mental em hospitais gerais, inclusão na RAPS e qualificação dos hospitais psiquiátricos especializados; a utilização das Comunidades Terapêuticas; e a criação de nova modalidade de Centro de Atenção Psicossocial de Álcool e Outras Drogas do tipo IV (CAPS-AD-IV)22.

Conforme exposto, a assistência à saúde mental brasileira teve importantes avanços, principalmente sobre o fenômeno da loucura e a própria concepção ampliada de saúde no território ${ }^{23}$. Contudo, apesar destes avanços, os serviços substitutivos ainda enfrentam dificuldades e barreiras de cuidado quanto à inserção do tratamento/acompanhamento em saúde mental no território ${ }^{23}$

A Política Nacional de Saúde Mental15 reforça a reorientação da atenção no território e a corresponsabilização do cuidado como forma de garantia de acesso universal, integral e qualificado aos usuários. Dessa forma, a APS é apontada como orientadora/coordenadora do cuidado na rede de atenção à saúde. Destacando-se que a reorientação do modelo tradicional de atenção à saúde está relacionada à adoção de práticas que tenham como premissa o acolhimento dos usuários ${ }^{5}$.

Assim, a inclusão da saúde mental na APS contribui para promover a ruptura com o modelo hospitalocêntrico, além de evidenciar a importância dos serviços substitutivos - como os CAPS de circularem mais pelo território, ressaltando a necessidade desses como um modus operandi, para além das estruturas físicas10.

Essa concepção de atenção propicia a redução do estigma em relação à loucura, além de ampliar o acesso da população aos cuidados em saúde mental, uma vez que a APS é o ponto do sistema que está mais próximo da vida das pessoas, tornando-se um ponto estratégico para a construção de novos modelos e práticas de atenção 5 .

A inclusão da saúde mental na APS fortalece o cuidado, permite uma atenção compartilhada, de uma formação ampliada e de possibilidades concretas de rupturas e avanços para a clínica psicossocial. Além de atender, entre outros, os princípios da integralidade e de equidade defendidos pelo SUS, avançando para o processo de desinstitucionalização16, no qual a assistência à saúde das pessoas ocorre onde elas vivem e com isso incentiva a sua comunidade à convivência com a loucura e a diferença, trabalhando a inclusão para que se possa minimizar os efeitos negativos do modelo biomédico que segrega e exclui ${ }^{5}$.

A APS passa a ser compreendida como ponto central do cuidado à saúde mental, por meio do desenvolvimento de estratégias e intervenções que possibilitem a configuração, desconfiguração 
e reconfiguração dos territórios existenciais individuais e coletivos. Apresenta a missão de deslocar o olhar da doença para o cuidado, para o alívio e a ressignificação do sofrimento e para a potencialização de novos modos individuais e grupais de estar no mundo, contribuindo, assim, para a efetivação da integralidade do cuidado, bem como as transformações requeridas como superação do modelo manicomial/excludente ${ }^{16}$.

Neste contexto, em uma Unidade Básica de Saúde (UBS), na cidade de Curitiba, criou-se um grupo de saúde mental para pacientes que tinham transtornos mentais graves, em sua área de abrangência. O grupo tinha como intuito acolher estes usuários e promover saúde na comunidade em que estão inseridos. Este grupo foi desenvolvido por acadêmicos do segundo período de Enfermagem, durante o curso da disciplina de Saúde Mental, de uma instituição particular de ensino parceira da UBS, sob a supervisão do professor da disciplina e das psicólogas do NASF.

As equipes de saúde da UBS indicaram os usuários com transtornos mentais graves para participar do grupo, o qual foi composto por 12 usuários com os seguintes diagnósticos: esquizofrenia com auto e hetero agressão; esquizofrenia paranoide; retardo mental moderado; transtorno depressivo recorrente; transtorno afetivo bipolar.

O grupo foi realizado na sala da Capela em frente à UBS, no período de março a novembro de 2017, tendo sua periodicidade semanal e duração média de 2 horas, contabilizando 30 encontros, nos quais foram realizadas diversas ações promotoras de convivência, incluindo atividades lúdicas, espaços de escuta, rodas de conversa, orientações/oficinas de saúde e psicoeducação. Além destas atividades, os alunos realizavam visitas domiciliares e busca ativa dos participantes (quando ocorria mais de duas faltas consecutivas no grupo ou em consultas); manejo verbal individual; troca de informações com as equipes da UBS, entre outras ações.

As estratégias utilizadas visavam fortalecer o vínculo entre o usuário e a UBS; melhorar a autoestima dos usuários; estimular e incentivar o autocuidado; monitorar e melhorar a eficiência do tratamento medicamentoso; contribuir para a estabilização dos quadros e minimizar o número de crises, internações e abandonos ao tratamento. Ademais, as intervenções tinham objetivo de romper com o modelo biomédico, favorecendo discussões sobre cada caso com os diferentes profissionais da equipe da APS (médicos, enfermeiros, dentistas, agentes comunitários de saúde, psicólogos, nutricionistas, educador físico, farmacêutico, etc.).

Pode-se perceber que tal iniciativa gerou mudanças na assistência em saúde mental na referida UBS, tanto para aquele que recebia o cuidado, quanto para o profissional que o realizava. Deste modo, considerou-se relevante a presente pesquisa, de modo a analisar e apresentar os resultados dessas ações de cuidado, a partir da percepção dos profissionais da UBS. Assim, o presente estudo definiu como objetivo investigar o efeito de um grupo de apoio à saúde mental a partir da percepção dos profissionais da Atenção Primária à Saúde.

\section{METODOLOGIA}

O presente estudo configura uma pesquisa exploratória descritiva com utilização dos métodos qualitativo e quantitativo, a partir da análise de conteúdo, com fundamentos em Bardin $^{24}$. As ocorrências dos termos dos discursos foram enumerados (quantitativo) e o conteúdo expresso foi analisado, objetivando a compreensão (qualitativo). Para tanto foi elaborado um instrumento de pesquisa semiestruturado para a coleta das percepções dos profissionais acerca do efeito do grupo de saúde mental no cuidado em saúde mental na APS.

Os critérios de inclusão na pesquisa foram: estar lotado na UBS selecionada; acompanhar o usuário(s) com transtorno mental grave que participava do grupo de saúde mental no território da UBS; ter interesse em participar da pesquisa, mediante a assinatura do Termo de Consentimento Livre e Esclarecido (TCLE). Para evitar viés ou 
inferências nos dados, foram excluídos da pesquisa as duas psicólogas, os acadêmicos e o docente de enfermagem que contribuíram para a realização das atividades do grupo. Também foram excluídos os profissionais que estiveram ausentes no período da coleta de dados, por desligamento do serviço, licenças ou férias; e ainda, aqueles que não quiseram participar.

Para a manutenção do sigilo dos participantes, formulou-se um instrumento autoaplicável, dispensando a presença de entrevistador, evitando inferências no preenchimento. Na entrega do questionário, também foi disponibilizado um envelope não identificado, para que este fosse devolvido ao pesquisador lacrado.

Os questionários foram numerados (Q1, Q2, Q3, etc.) a fim de preservar a identidade dos participantes na tabulação e análise de dados. 0 instrumento semiestruturado foi composto por cinco questões fechadas (idade, sexo, nível de escolaridade, profissão, tempo de atuação na unidade de saúde) e sete questões abertas: 1) Quais são os usuários que estão inseridos no grupo de saúde mental da UBS que você acompanha? Há quanto tempo você conhece estes pacientes?; 2) Qual a forma de cuidado / relação estabelecida entre você e o participante do grupo? ; 3) Você percebeu alguma alteração nesta relação de cuidado desde que o usuário começou a participar do grupo de saúde mental?; 4) Como você descreve/caracteriza os pacientes que estão inseridos no grupo de saúde mental?; 5) Quais as dificuldades e facilidades que você percebe no acompanhamento destes usuários na UBS?; 6) Você percebeu mudanças nos usuários que você acompanha após a participação destes no grupo de saúde mental ofertado pela UBS? Descreva as mudanças observadas; 7) Qual a sua opinião a respeito da estratégia grupo de saúde mental como parte do tratamento de saúde mental na APS?

Quanto aos cuidados éticos, a pesquisa foi aprovada pelo Comitê de Ética da Secretaria Municipal de Saúde de Curitiba, por meio do protocolo de número 2.295.314, o que atendeu à normativa de pesquisa envolvendo seres humanos.
Para a análise qualitativa de resultados utilizou-se o método Análise de Conteúdo, com base na Análise Temática de Bardin ${ }^{24}$, composto por três fases: (1) pré-exploração, caracterizada pela seleção do corpus a ser analisado, sendo realizada a leitura de todo material, apreendendose os aspectos relevantes; (2) seleção das unidades de análise coerentes aos objetivos da pesquisa; (3) categorização e subcategorização dos dados selecionados, sendo classificados em temas, conforme grau de intimidade ou proximidade, e expressão de significados e elaborações que atenderam aos objetivos do estudo e criaram novos conhecimentos, proporcionando uma visão diferenciada sobre os temas propostos ${ }^{24}$.

\section{RESULTADOS E DISCUSSÃO}

Participaram da pesquisa 13 profissionais, 5 de nível superior (médico, enfermeiro e nutricionista); 5 técnicos e auxiliares de saúde (enfermagem e saúde bucal); e 3 agentes comunitários. Esses profissionais desenvolvem suas atividades na unidade de saúde através da Estratégia Saúde da Família, cujos usuários, diagnosticados com algum transtorno mental, estão inseridos no Grupo de Saúde Mental do Curso de Enfermagem de uma Universidade particular de Curitiba.

Os profissionais entrevistados tinham em média 45 anos de idade. 0 tempo de atuação na unidade de saúde varia entre 1 e 18 anos, com média de 9 anos de atuação no território. Em média os participantes acompanham entre 2 e 3 usuários inseridos no grupo, há um profissional que acompanha 1 usuário e um que acompanha 6.

Por meio da análise do discurso dos profissionais, podem-se observar diversos elementos que permeiam a assistência em saúde mental na APS. Desta forma, os relatos foram organizados em temas que estão agrupados em cinco núcleos de discussão: (1) relação profissional/ usuário na APS; (2) percepção sobre o perfil 
dos usuários diagnosticados com transtorno mental; (3) percepção sobre as potencialidades e dificuldade na assistência; (4) a percepção sobre a intervenção; e (5) a percepção sobre as mudanças de comportamento dos usuários.

Ao analisar o primeiro núcleo, a relação profissional/usuário na APS (Figura 1), foi possível destacar 5 temas: atendimento; cuidado com a medicação; integralidade do cuidado; vínculo; e acolhimento.

Em 27\% das respostas o atendimento mostrou-se como uma das características da relação entre o participante e o usuário. Percebese que esta relação ainda está atrelada ao modelo biomédico, no qual o agente de saúde presta serviços "mecanicistas" como: agendamentos, entrega e/ou administração de medicamentos, consultas, etc. Essa característica também pode ser percebida através da preocupação com a medicação dos usuários, presente em 24\% dos relatos, sendo consequência e, concomitantemente, estimulada pela hegemonia do modelo biomédico ${ }^{25}$.

Figura 1. Percepção sobre a relação entre os usuários e os profissionais da APS

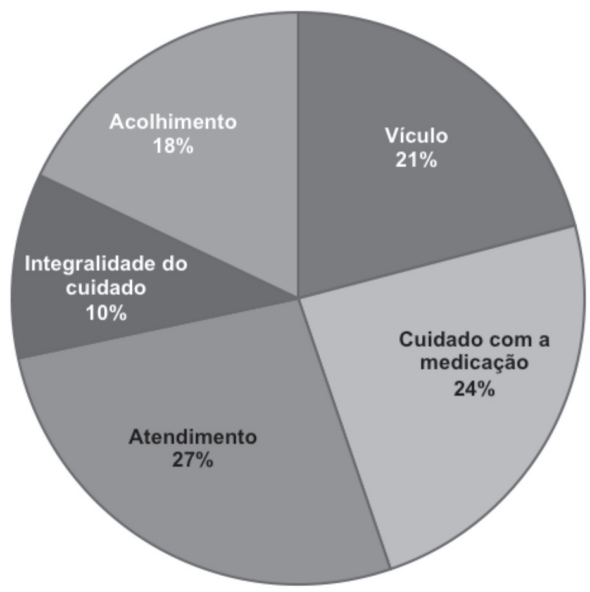

Fonte: Elaborado pelos autores (2018)
Neste sentido, há uma representação do processo saúde-doença em que a relação é mediada por um pensamento/desejo de agir sobre algo, exercendo um domínio sobre o outro, sobre um signo/significado ${ }^{25}$. Enxergar a relação como quem a prescreve, entrega e/ou administra a medicação é assumir uma posição ativa, de quem interfere nos sintomas, em detrimento a uma posição passiva (paciente), daquele que recebe o cuidado, sendo que se espera uma postura inversa a esta, no sentido de se promover a autonomia do usuário, visando seu autocuidado.

Concomitante a esta observação, alguns relatos apresentam características que destoam do modelo biomédico, sendo organizados nos temas: vínculo (21\%), acolhimento (18\%) e integralidade do cuidado (10\%). Essas relações se aproximam da ideologia defendida pelo modelo biopsicossocial - que proporciona uma visão integral do ser e do adoecer, compreendendo as dimensões física, psicológica e social -, que temse afirmado progressivamente e é considerado como uma alternativa ao modelo biomédico ${ }^{26}$, pois afirma que a relação de cuidado deve ser baseada no acolhimento, no vínculo e na integralidade da atenção. Como pode ser observado nos relatos a seguir: "a nossa relação é com cordialidade e paciência" (Q7); "Se dô super bem, tem carisma muito grande com eles" (Q11); e "saber ouvir e entender as limitações"(Q13).

Nesse modelo o destaque para o cuidado são as tecnologias leves ${ }^{27}$, tecnologias de relação, que demonstram serem fundamentais na produção do cuidado principalmente no acolhimento, formação de vínculo e acesso, levando à autonomia.

No segundo núcleo de discussão, percepção sobre o perfil dos usuários diagnosticados com transtorno mental (Figura 2), os participantes descreveram os usuários com as seguintes características: carentes, depressivos; ansiosos, dependentes, irritados, excluidos socialmente, apoio familiar frágil, comprometimento psíquico (comprometimento cognitivo, afetivo, mentais, especiais) e de fácil vínculo. 
Figura 2. Percepção sobre o perfil dos usuários

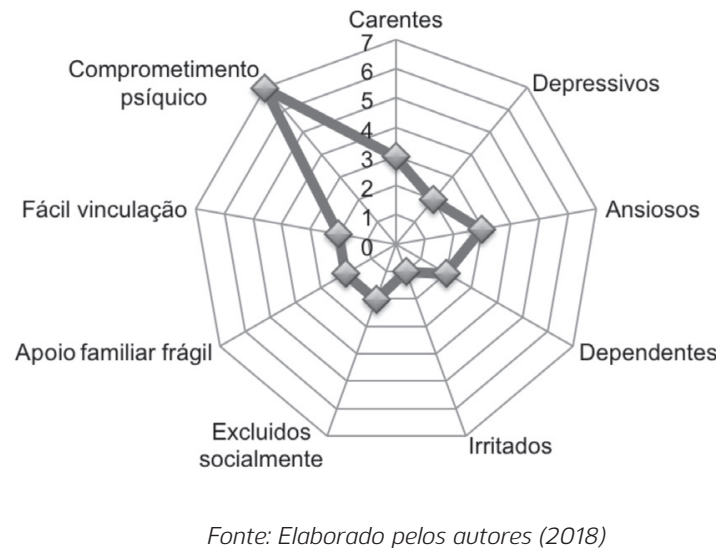

Conforme verificado, os profissionais consideram os pacientes como carentes, dependentes e irritados, essas características evidenciam que os transtornos mentais ainda estão associados a estereótipos negativos que contribuem nas barreiras de acesso7. que limitam a produção de saúde, por uma crença de que os pacientes com transtorno mental não são capazes de assumir compromisso com o tratamento. Desta forma, pode-se inferir que mesmo com um olhar diferente do senso comum, oportunizado pela formação profissional, que sensibiliza para atuar com este público, por meio da informação técnica e científica, que as representações sociais da loucura e a influência cultural cristalizam estereótipos, interferindo desta forma na relação de cuidado e no acesso.

Na categoria "percepção sobre as dificuldades e potencialidades na assistência" os profissionais apontam o desfalque da própria equipe de saúde, o tempo limitado nos atendimentos, dificuldade de monitorar os pacientes, falta de apoio familiar/ comunitária e o problema de compreensão da queixa do usuário como as dificuldades que enfrentam no atendimento dos usuários. Destacam, ainda, a falta de adesão dos usuários aos tratamentos ofertados nos CAPS.

Entretanto, em relação às potencialidades que facilitam o atendimento dos usuários, ressaltase o apoio das instituições de ensino, a adesão e participação nas consultas e atividades propostas na Unidade Básica de Saúde, a facilidade de formar vínculo e demonstrar afeto.

Figura 3. Percepção sobre as dificuldades na assistência

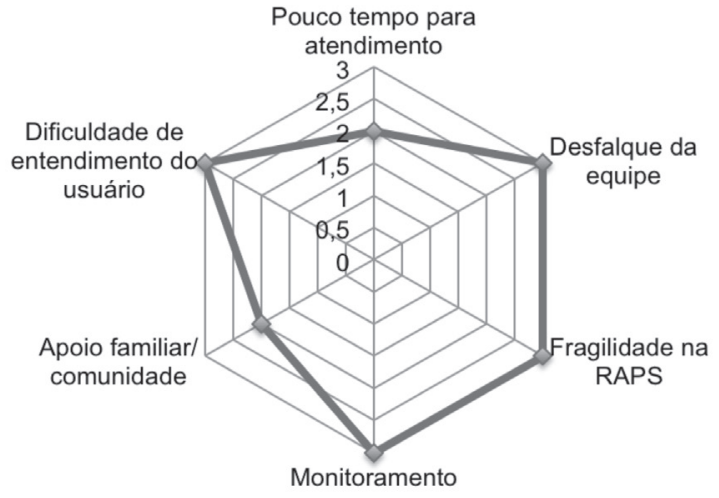

Fonte: Elaborado pelos autores (2018)

Figura 4. Percepção sobre as potencialidades na assistência

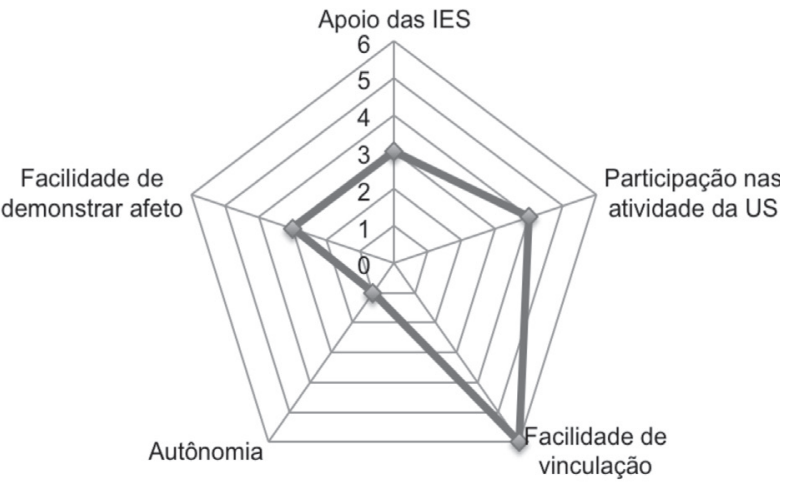

Fonte: Elaborado pelos autores (2018)

Por fim, nas palavras dos participantes da pesquisa, a intervenção foi avaliada como: "uma ferramenta para a qualidade de vida (melhora) dos pacientes inseridos" (Q9) que serviu "como apoio para a equipe da unidade de saúde, diminuindo as intervenções" (Q4)... e contribuindo no "sucesso do tratamento do paciente, vínculo paciente/ funcionário, entendimento do contexto em que vive o paciente" (Q2), considerando "uma forma 
deles (usuários) se sentirem mais úteis e integrados na comunidade" (Q7), "eles gostaram e melhoraram a autoestima" (Q12).

Em relação à percepção dos profissionais quanto às alterações do comportamento dos usuários (Figura 5), após a intervenção, observaramse cinco temas principais: vínculo; autonomia; comunicação; adesão ao tratamento; e autoestima.

Figura 5. Percepção sobre as potencialidades na assistência

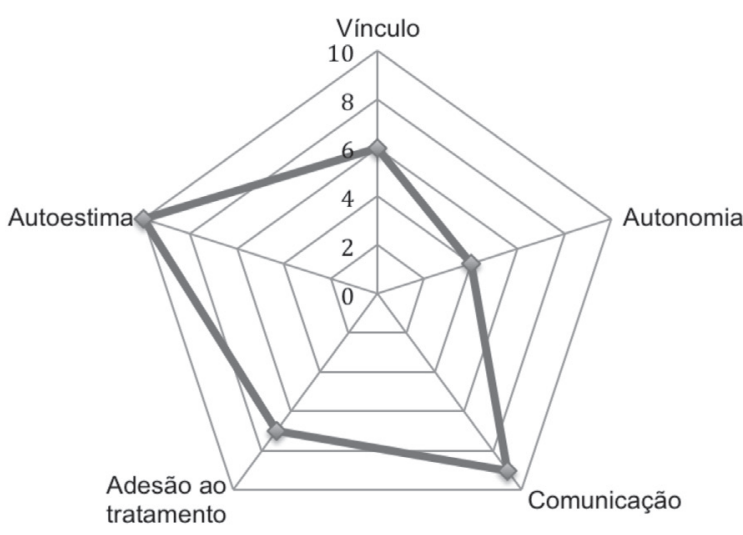

Fonte: Elaborado pelos autores (2018)

Perceberam mudança na autoestima dos usuários, observando maior motivação e animação no dia a dia, e relatam que os pacientes se sentem valorizados. Outra mudança observada foi a forma de se comunicar com a Unidade de Saúde, na qual os usuários se apresentaram com uma postura mais autônoma e participativa, respeitando os seus limites e dos outros, tornando-se mais acessiveis e comunicativos.

A percepção dos profissionais, concernente aos usuários, ressalta uma ambivalência. Há um receio (medo) daquele que pode perder o controle de suas emoções, de seus impulsos e comportamento, ao mesmo tempo em que revelam a existência de afeto e respeito. Outro tópico ambíguo é o reconhecimento e a observação da autonomia dos usuários, ao mesmo tempo em que os caracterizam como pessoas dependentes.

\section{CONSIDERAÇÕES FINAIS}

No presente estudo o vínculo foi o tema que se mostrou presente em todos os núcleos de discussão. Pode-se concluir que o tempo de atuação no território, que em média é de 9 anos, contribui na longitudinalidade do cuidado. O vínculo longitudinal é um dos atributos da Atenção Primária à Saúde. considerando-o como uma característica central e exclusiva deste nível de atenção, conceituando como o acompanhamento realizado ao usuário ao longo do tempo por uma equipe de saúde da família para os múltiplos episódios de doença e cuidados preventivos $^{28}$.

O vínculo, então, favorece o cuidado integral, à medida em que estabelece uma relação afetiva, de confiança, respeito e a valorização entre a unidade de saúde e a comunidade em que está inserida. Desse modo, a percepção dos profissionais da APS contribui para avaliação das intervenções realizadas com os usuários do seu território.

Perante o exposto, evidencia-se que o cuidado em saúde mental é caracterizado por um processo de elaboração das crenças preexistentes, como um novo saber que norteia o cuidado, saber este que se atualiza por meio das relações de vínculo e afeto, onde usuários e profissionais transformamse uns aos outros, através do convívio, respeito e da humanização.

Porém, para que se possa avançar na assistência em saúde mental é de suma importância entender que a saúde mental no Brasil vem sofrendo rápidas mudanças, que por diversas vezes são inovadoras e contribuem para o cuidado mais humanizado, mas que por vezes aponta para o retrocesso. Debruçar-se sobre este fenômeno contribui para que possamos repensar nos processos de trabalho, refletir sobre ações que contribuem para as barreiras de acesso, ou para a culpabilização do usuário pela não adesão ao serviço. De forma que lidar com a dificuldade do outro é encarar a própria dificuldade do profissional, que pode transcender questões técnicas e políticas, atingindo sua própria subjetividade. 
Por fim, evidencia-se que as práticas ainda são embasadas em uma forte visão biomédica, minimizando-se a história e individualidade dos sujeitos, sendo estas ações de cuidado reforçadas pelo raciocínio anatomoclínico e fisiopatológico, lógico, baseado em evidências científicas16. Faz-se necessário que os profissionais reconheçam que as demandas de saúde mental estão presentes em diversas queixas relatadas pelos usuários que chegam aos serviços de saúde, independente se há um diagnóstico ou não. Assim, os profissionais poderão ofertar assistência de forma mais integral, sem fragmentar a mente e o corpo, não dissociando a saúde emocional da saúde geral.

Desta forma, se mostra de extrema importância e de grande valia a inclusão de ações de saúde mental na APS, a fim de sensibilizar os demais profissionais de saúde e facilitar a incorporação e o aprimoramento de competências de cuidado em saúde mental prática diária, de tal modo que suas intervenções sejam capazes de considerar a subjetividade, a singularidade e a visão de mundo do usuário no processo de cuidado integral à saúde ${ }^{16}$.

\section{REFERÊNCIAS}

1. Amarante PDC. Reforma Psiquiátrica e Epistemologia. Cad. Bras. Saúde Mental, vol.1, n.1, jan-abr, 2009.

2. Prado Filho K, Lemos FCS. Uma breve cartografia da luta antimanicomial no Brasil. Contemporânea - Revista de Sociologia da UFSCar, São Carlos, v. 2, n. 1, p. 45-63, jan-jun, 2012.

3. Amarante PDC. Psiquiatria social e reforma psiquiátrica. Rio de Janeiro, Fiocruz, 1994

4. De Leon G. Comunidade Terapêutica - teoria, modelo e método. Ed. Loyola, São Paulo, SP, 2003

5. Souza AC. Estratégias de Inclusão da Saúde Mental na Atenção Básica - Col. Saúde Loucura. Ed. Hucitec, 2015

6. Amarante PDC. O homem e a serpente: outras histórias para a loucura e a psiquiatria. Rio de Janeiro, Fiocruz, 1996.

7. Organização Mundial da Saúde - Saúde Mental: nova concepção, nova esperança: relatório sobre a saúde no mundo. Geneve: OMS, 2001.
8. Brasil, Ministério da Saúde. Secretaria de Atenção à Saúde. Saúde Mental e Atenção Básica: o vínculo e o diálogo necessários - Inclusão das ações de Saúde Mental na Atenção Básica. Brasília: Ministério da Saúde; Circular Conjunta n. 01 e 03, 2003.

9. Declaração de Alma-Ata. Conferência Internacional sobre Atenção Primária à Saúde. Alma-Ata, 1978.

10. Santos Y. O ambulatório de saúde mental no contexto da reforma psiquiátrica em Natal/RN. Dissertação de mestrado, Universidade Federal do Rio Grande do Norte, Rio Grande do Norte, 2007.

11. Yasui S. Rupturas e encontros: desafios da reforma psiquiátrica brasileira. Tese de Doutorado, Curso de Pós-graduação em Saúde Pública, Escola Nacional de Saúde Pública, Rio de Janeiro, Fiocruz, 2006.

12. Antonacci MH, Pinho LB. Saúde Mental na Atenção Básica: uma abordagem convergente assistencial. Rev Gaúcha Enferm, Porto Alegre, 2011

13. Neto FK. Reforma psiquiátrica e conceito de esclarecimento: reflexões críticas. Rev. Mental - Ano I - n. 1 - Barbacena, dez, 2003.

14. Brasil. Ministério da Saúde. Secretaria Executiva. Secretaria de Atenção à Saúde. Legislação em saúde mental: 1990-2004. 5a ed. ampl. Brasília: Ministério da Saúde, 2004.

15. Brasil. Lei 10.216 de 6 de abril de 2001. In: Brasil. Ministério da Saúde. Secretaria de Atenção à Saúde. Legislação em Saúde Mental:1990-2004. 5ª ed. Brasília: 2004

16. Brasil. Ministério da Saúde. Cadernos de Atenção Básica, n. 34 Saúde Mental. Brasília: Ministério da Saúde, 2013.

17. Jorge MSB, Randemark NFR, Queiroz MVO, Ruiz EM Reabilitação Psicossocial: visão da equipe de Saúde Mental. Rev Bras Enfermagem, Fortaleza, v. 59, n.6. p.734-739, nov./dez. 2006

18. Mendonça RT, Carvalho ACD, Vieira EM, Adorno RCF Medicalização de mulheres idosas e interação com consumo de calmantes. Saúde Soc., p. 95- 106, 2008

19. Brasil. Ministério da Saúde. Gabinete do Ministro. Portaria $n^{\circ}$ 154, de 24 de janeiro de 2008. Cria os Núcleos de Apoio à Saúde da Família - NASF. Diário Oficial da República Federativa do Brasil, Brasilia, 2008.

20. Brasil. Ministério da Saúde. Cadernos de Atenção Básica, n. 27. Diretrizes do NASF: Núcleo de Apoio à Saúde da Família. Brasília: Ministério da Saúde, 2010

21. Brasil. Portaria GM 3088, de 23 de dezembro de 2011. Institui a Rede de Atenção Psicossocial para pessoas com sofrimento ou transtorno mental e com necessidades decorrentes do uso de crack, álcool e outras drogas, no âmbito do Sistema Único de Saúde (SUS) Diário Oficial da União, 2011.

22. Brasil. Portaria no 3.588, de 21 de dezembro de 2017. Altera as Portarias de Consolidação no 3 e no 6, de 28 de setembro de 2017, para dispor sobre a Rede de Atenção Psicossocial e dá 
outras providências. Diário Oficial da União - Seção 1, no 245, 22 de dezembro de 2017.

23. Heidrich AV. Reforma psiquiátrica à brasileira: Análise sob a perspectiva da desinstitucionalização. Tese de Doutorado - Porto Alegre: PUCRS, 2007

24. Bardin L. Análise de conteúdo. Lisboa: Edições $7^{a}$ Ltda, 1977.

25. Barros JAC. Pensando o processo saúde doença: a que responde o modelo biomédico? Rev Saúde Soc, 2002

26. Marco MA. Do modelo biomédico ao modelo biopsicossocial: um projeto de educação permanente. Rev Bras Educ Med, v.30, n.1, p.60-72, 2006.

27. Merhy EE. Em busca do tempo perdido: a micropolítica do trabalho vivo em saúde. In: Merhy EE, Onocko R. Agir em saúde: um desafio para o público. São Paulo: Hucitec; p.74-111, 1997.

28. Santos ROM. O vínculo longitudinal como dispositivo do cuidado: Saúde da Família e doenças crônicas em uma comunidade do Rio de Janeiro. Dissertação de mestrado, Escola Nacional de Saúde Pública Sergio Arouca, Rio de Janeiro, 2015. 